\title{
The Impact of Color Rendering on Visual Fatigue in Interior Zone of Tunnel
}

\author{
Peng Liu ${ }^{1}$, LiLi Dong ${ }^{1, *}$, and YingQi Jiang ${ }^{1}$ \\ ${ }^{1}$ School of Information Science and Technology, Dalian Maritime University, 116026 Dalian, China
}

\begin{abstract}
Judicious use of lamps is of profound significance to improve the internal traffic safety of tunnels. This study evaluated the effect of LED color on human visual fatigue under mesopic vision category. According to the difference of human eyes' response to different wavelengths of light radiation, the mesopic vision spectral luminous efficiency curve is applied to the visual fatigue evaluation methods. Taking the critical fusion frequency as the physiological index, the detection experiment of human visual fatigue was carried out in the simulated tunnel environment. The results show that spectrum with high color rendering index has a positive effect on alleviating drivers' visual fatigue, and is more suitable for tunnel interior lighting.
\end{abstract}

\section{Introduction}

Visual fatigue is one of the important causes of traffic accidents. Due to the semi-enclosed space characteristics of highway tunnels, its visual pressure and monotonous environment are more likely to aggravate the visual fatigue, which seriously affects road traffic safety[1]. Therefore, it is imperative to study tunnel lighting from the perspective of visual fatigue.

In recent years, light emitting diode(LED) has been widely used in traffic lighting due to its long life, energy saving and other advantages. LED has adjustable spectrum, which makes it possible for people to choose the light color of LED. How to select the light color according to the needs of the scene has become an urgent problem to be solved. At present, there is no clear basis for the selection of light color in the tunnel lighting design department.

At present, the LED used in road lighting is made of yellow phosphor excited by blue light, which makes the blue light content of the spectrum higher. Due to the short wavelength of blue light, the focus point does not fall in the center of the human retina, but a little more in front of the retina. If you want to see clearly, the eyeball will be in a state of tension, causing visual fatigue and affecting people's driving performance. How to choose the appropriate light color parameters to reduce the harm of blue light and alleviate the driver's visual fatigue needs to be studied.

Hawes et al. compared the effects of fluorescent lamp and LED lamp on cognitive performance and visual fatigue, and the results showed that the fatigue degree of subjects under fluorescent lamp was higher than that of LED[2]. Wang Qing et al. studied the effect of LED on visual fatigue based on reading task, and the results showed that compared with low illumination and low correlated color temperature(CCT), the symptoms of visual fatigue in high illumination and high CCT were lighter[3]. Liang Bo et al. studied the influence of light source CCT on reaction time, and the results showed that increasing light source CCT can improve the visual efficiency[4].

At present, most of the researches on visual fatigue focus on light intensity and CCT, but the research on color rendering index $(\mathrm{CRI})$ is still lacking. Therefore, this paper studies the influence of CRI on visual fatigue in low visibility. This paper aimed to select the light color parameters which are more conducive to alleviate the driver's visual fatigue, so as to reduce the occurrence of traffic accidents. The research results can provide reference for tunnel lighting standards, which is of great significance to improve road safety.

\section{Theoretical calculation}

In recent years, the blue light hazard factor $\mathrm{K}_{\mathrm{b}}$ of light radiation has been used to quantitatively reflect the influence of different wavelengths on the blue light hazard of human eyes. The higher the value, the greater the impact on visual fatigue. It can be calculated by equation (1).

$$
K_{b}=\frac{\int_{380}^{780} L_{e d}(\lambda)^{*} B(\lambda) \mathrm{d} \lambda}{K_{e} \int_{380}^{780} L_{e d}(\lambda)^{*} E(\lambda) \mathrm{d} \lambda}
$$

where $\mathrm{L}_{\mathrm{ed}}(\lambda)$ refers the SPD of the light source. Huai et al. studied the influence of three kinds of CCT on the penetration characteristics of fog, and obtained that $3000 \mathrm{~K}$ is more suitable for street lighting by calculating the transmittance[5]. We chose the spectra with different $\mathrm{CRI}(60,70,80$ and 90$)$ at $3000 \mathrm{~K}$ for experimental study, which was shown in Figure 1 (a). B( $\lambda$ ) refers the spectral

* Corresponding author: donglili@dlmu.edu.cn 
weighting function of retinal blue light damage, which was shown in Figure 1 (b). $E(\lambda)$ is the spectral luminous efficiency function of human vision, and $\mathrm{K}_{\mathrm{e}}$ is its maximum spectral luminous efficiency. Because the tunnel lighting section belongs to the category of mesopic vision, it is unreasonable to use the human visual curve under photopic vision. Therefore, this paper uses the MES-2 model to obtain mesopic vision spectral light curve. Previous studies show that the brightness of this model (below $5.0 \mathrm{~cd} / \mathrm{m}^{2}$ ) is more suitable for tunnel lighting[6]. The form of MES-2 mesopic vision spectral luminous efficiency model is as follows:

$$
\begin{gathered}
L_{S}=K_{\mathrm{m}}^{\prime} \int_{380}^{780} V^{\prime}(\lambda) L_{e d}(\lambda) \mathrm{d} \lambda \\
L_{P}=K_{m} \int_{380}^{780} V(\lambda) T(\lambda) L_{e d}(\lambda) \mathrm{d} \lambda \\
L_{m e s, n}=\frac{m_{2,(n-1)} L_{P} K_{\mathrm{m}}^{\prime}+\left(1-m_{2,(n-1)}\right) L_{S} K_{m}}{m_{2,(n-1)} K_{\mathrm{m}}^{\prime}+\left(1-m_{2,(n-1)}\right) K_{m}}, m_{2,0}=0.5 \\
m_{2, n}=0.3334 \log L_{m e s, n}+0.767,0 \leq m_{2, n} \leq 1 \\
V_{\text {mes }}(\lambda)=\frac{m_{2, \text { end }} V(\lambda)+\left(1-m_{2, \text { end }}\right) V^{\prime}(\lambda)}{\frac{m_{2, \text { end }}}{K_{m}}+\frac{\left(1-m_{2, \text { end }}\right)}{K_{\mathrm{m}}^{\prime}}}
\end{gathered}
$$

where $V^{\prime}(\lambda)$ refers the scotopic spectral luminous efficiency function, and $K_{m}{ }^{\prime}$ refers its peak value. $V(\lambda)$ refers the photopic spectral luminous efficiency function, and $\mathrm{K}_{\mathrm{m}}$ is its peak value. $\mathrm{T}(\lambda)$ refers the transmittance of different wavelength at a certain fog concentration, which was shown in Figure 1 (c). $\mathrm{L}_{\mathrm{S}}$ is the scotopic luminance, $\mathrm{L}_{\mathrm{P}}$ is the photopic luminance. (4) and (5) are iterative processes, $\mathrm{n}$ is an iteration step, $\mathrm{m}_{2 \text {,end }}$ is the final iteration output. $\mathrm{V}_{\text {mes }}(\lambda)$ is the mesopic spectral luminous efficiency function, which was shown in Figure $1(d)$. In the calculation, we use the product of $\mathrm{T}(\lambda)$ and $\mathrm{V}_{\text {mes }}(\lambda)$ as $E(\lambda)$.

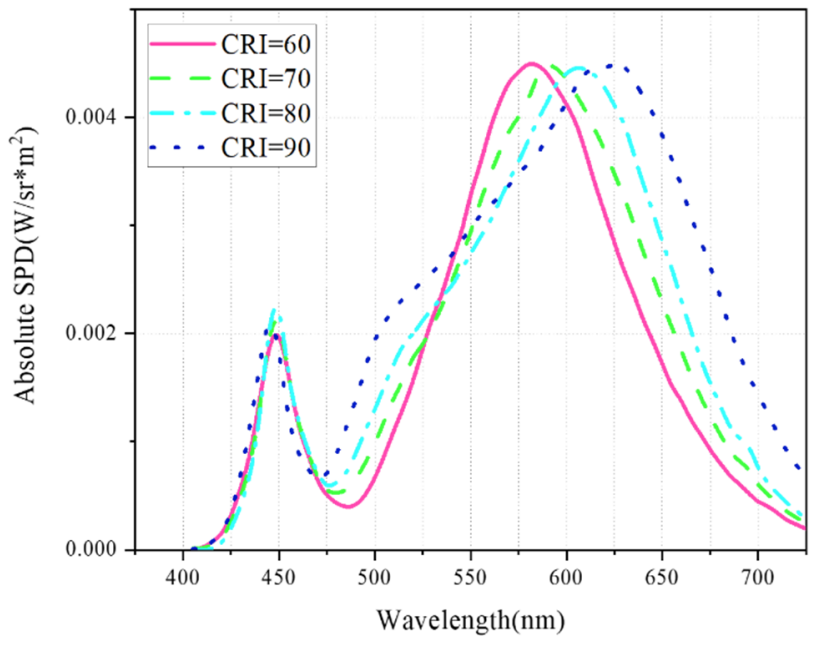

(a)

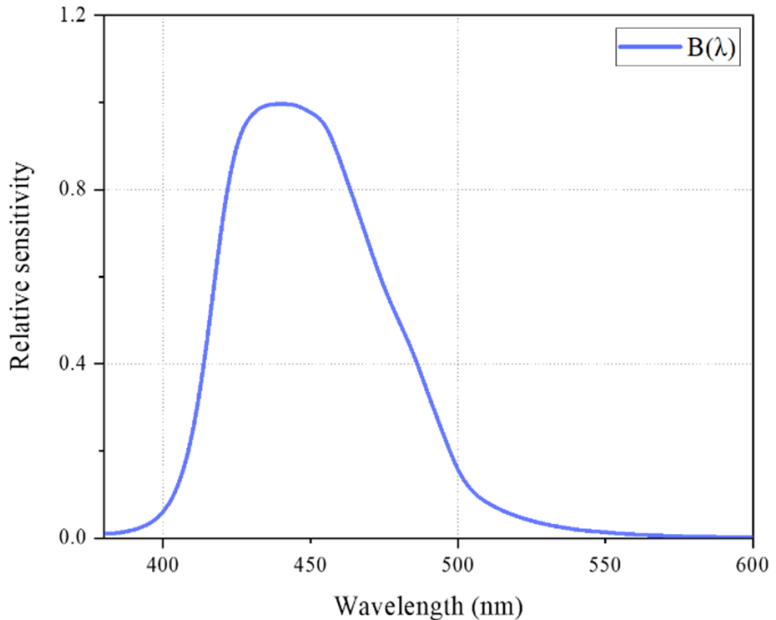

(b)

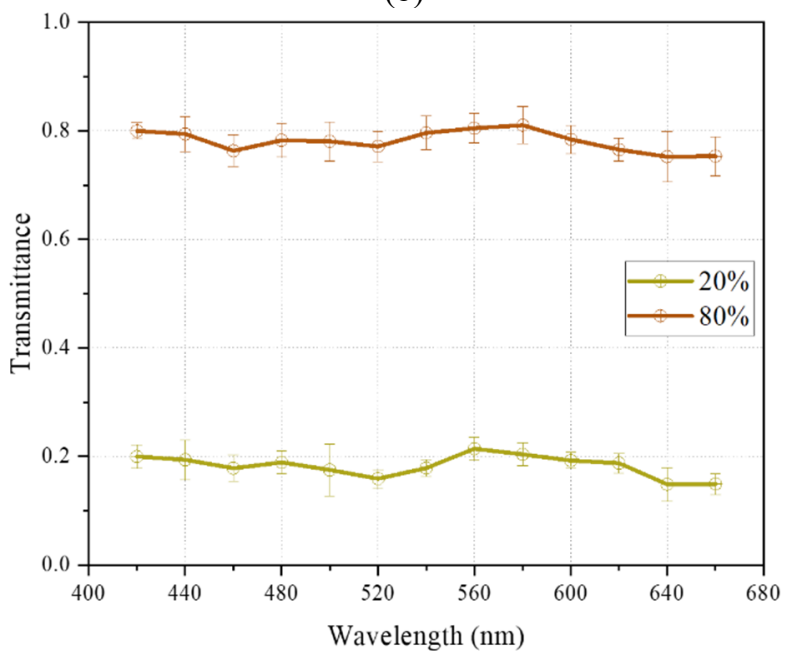

(c)

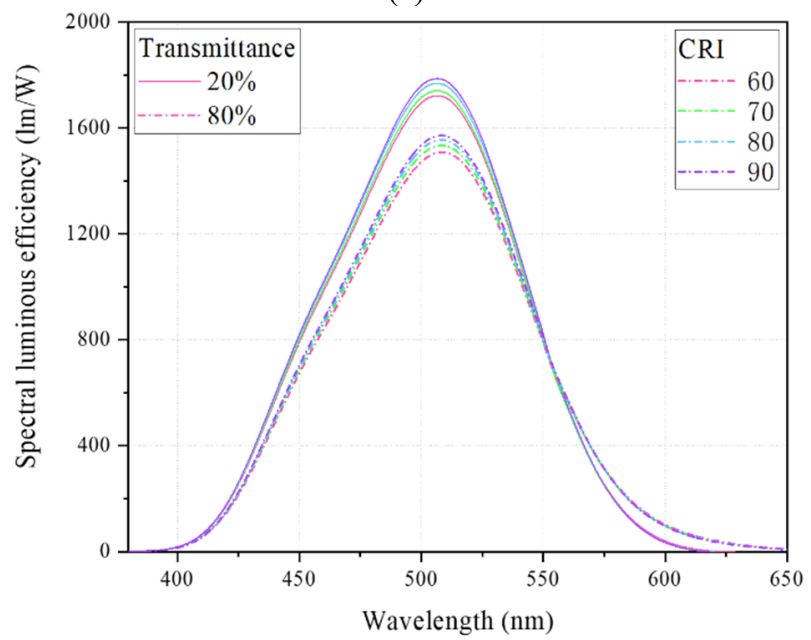

(d)

Figure 1. The curves of the parameters in equation (1) (6). (a) $\mathrm{L}_{\mathrm{ed}}(\lambda)$. (b) $\mathrm{B}(\lambda)$. (c) $\mathrm{T}(\lambda)$ and $(\mathrm{d}) \mathrm{V}_{\text {mes }}(\lambda)$.

Figure 2 shows the blue light hazard factors of four CRI $(60,70,80,90)$ spectra under mesopic vision when the fog transmittance is $20 \%$ and $80 \%$ respectively. It can be seen that the blue light hazard factor at $20 \%$ transmittance is greater than that at $80 \%$ transmittance. As for the effect of CRI on the blue light hazard factors, the blue light hazard factors decreased with the increase of CRI. From the theoretical calculation results, it can be preliminarily judged that the use of high CRI light source 
is helpful to driver's visual fatigue and improve road traffic safety.

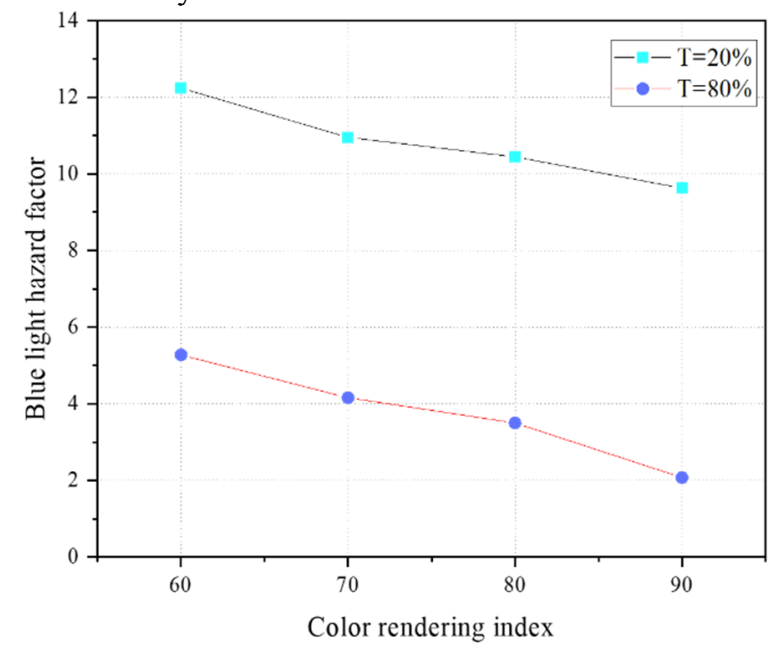

Figure 2. Blue light hazard factor.

\section{Experiment and results}

\subsection{Experimental setup}

According to the above theoretical analysis, we conclude that high CRI light source under mesopic vision is helpful to alleviate visual fatigue. However, in the actual tunnel driving environment, the influence of different CRI spectra on visual fatigue still needs further research. As an organ that directly feels light stimulation, the change of eye activity can most directly reflect visual fatigue. Previous studies have confirmed that the decline of critical fusion frequency(CFF) reflects the weakening of human eye function, which can be used to measure the degree of human visual fatigue[7]. The lower the CFF value, the greater the fatigue degree[8]. Compared with other physiological indicators, CFF is more simple and accurate in measuring asthenopia. In this paper, CFF is selected as the physiological parameter to detect visual fatigue.

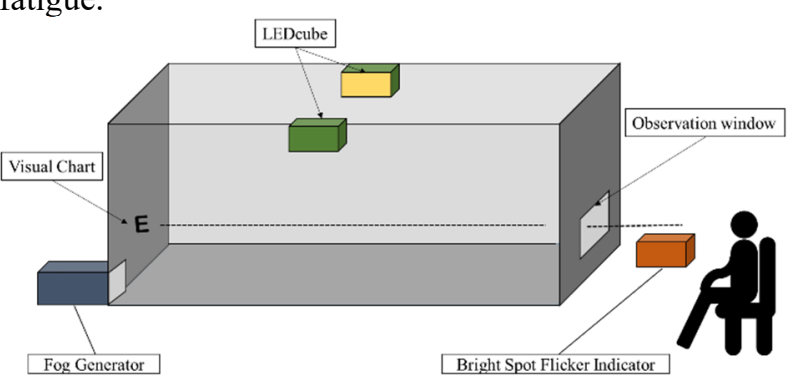

Figure 3. The schematic diagram of experimental set-up.

Figure 3 shows a schematic of the experimental setup. The fog chamber is made of plexiglass with a size of $3 *$ $2 * 2$ cubic meters. The inside of the glass box was pasted with wallpaper imitating the road surface and walls of the tunnel. LEDcubes are placed on both sides of the glass box to simulate different SPDs and light intensity. An observation window is set on the side close to the observer, and the bright spot flicker indicator is placed on the observer's chest for CFF measurement. A white visual chart " $E$ " is placed directly in front of the observer. The fog generator is placed behind the fog chamber to produce stable and uniform fog. Ten subjects with normal color vision and normal vision (or corrected vision) participated in the experiment.

First, the fog generator emits the fog until the fog concentration of the glass box reaches the required value ( $20 \%$ or $80 \%$ ). At the same time, the LEDcube is adjusted to the required light environment (CRI set to 60, 70, 80, 90). The luminance is set to $3 \mathrm{~cd} / \mathrm{m}^{2}$, which corresponding to the actual luminance inside the tunnel. Then the subjects were asked to measure CFF before the experiment. The frequency of CFF measurement was from low to high once, and from high to low again. The CFF value recorded by the experimenter is the average of the two measurements. Then the subjects were asked to focus their eyes on the opposite visual chart. CFF values were measured at 5,10 and 15 minutes of the experiment.

\subsection{Results and discussion}

The CFF values of 10 subjects under different spectral conditions were obtained by the experimental procedure. In the experiment, the CFF value of 0 minutes of each experiment is taken as the initial value, and the initial value minus the CFF value of 5 minutes, 10 minutes and 15 minutes is taken as the CFF drop value. Figure 4 shows the CFF drop values for different CRI sources at $20 \%$ and $80 \%$ transmittance.

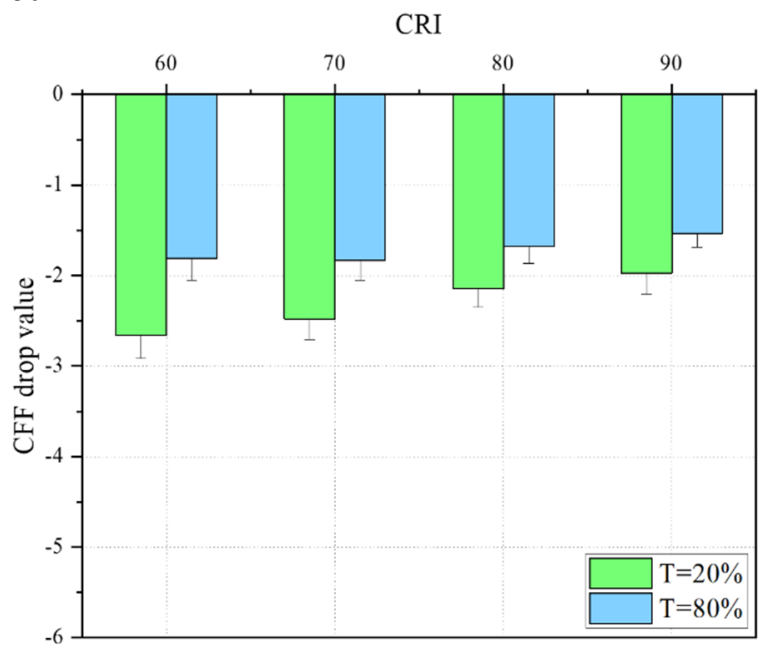

(a)

CRI

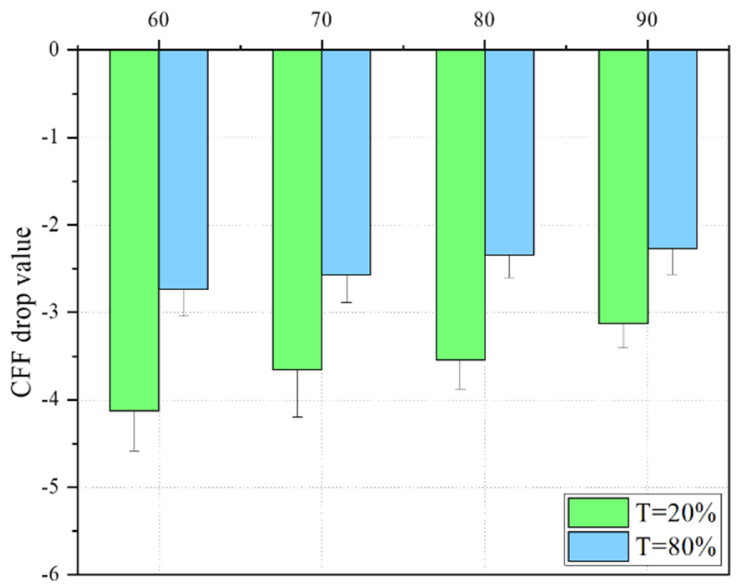

(b) 


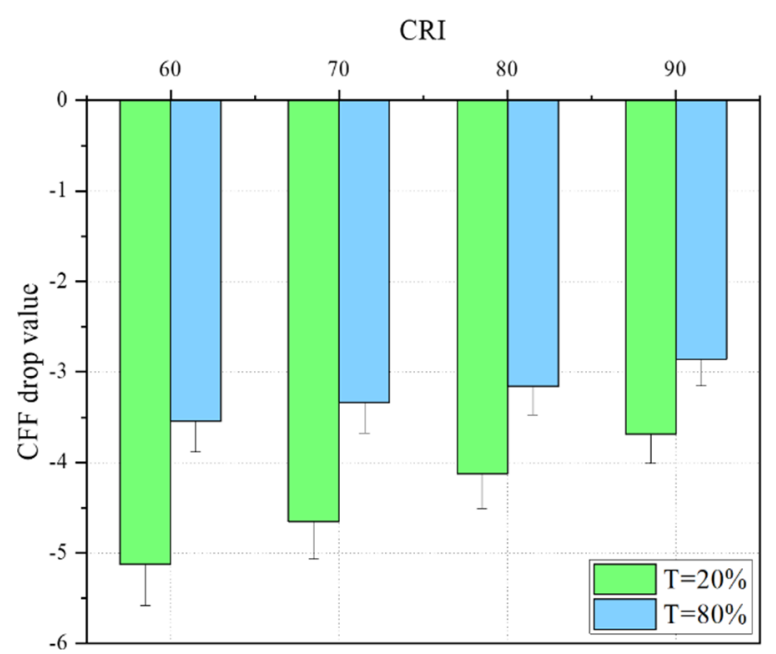

(c)

Figure 4. Average CFF drop value.

It can be seen from Figure 4 that CFF drop value increases with the increase of time, and decreases with the increase of CRI and fog transmittance. This shows that reducing the fog concentration inside the tunnel and improving the CRI of the light source are helpful to alleviate the driver's visual fatigue.

\section{Conclusions}

This paper discusses the effect of CRI on human visual fatigue under the condition of mesopic vision and low visibility. Firstly, a theoretical calculation model is established by combining the mesopic vision curve with the blue light hazard factor curve. The theoretical results show that high CRI has a positive effect on alleviating driving fatigue. Then, a fog chamber system was established to simulate the internal driving environment of the tunnel, and the CFF of the simulated driver was tested. The results show that the CRI and the fog transmittance are inversely proportional to the CFF decrease. Given the fog transmittance and the mesopic vision, high CRI light source is recommended in the interior zone of the tunnel to alleviate the driver's visual fatigue and ensure the driving safety.

\section{Acknowledgements}

This paper was supported in part by the National Natural Science Foundation of China under Grant 61701069. This paper was supported in part by the Fundamental Research Funds for the Central Universities of China under Grant 3132019340 and 3132019200 .

\section{References}

1. Shy, Bassan. Iatss Research. 40, 35-46 (2016)

2. A. Bkh, D. Ttba, D. Crma, C. Jms, B. Cda. Int J Ind Ergonom. 42, 122-128 (2012)

3. W. Qing, X. Haisong, G. Rui, C. Jianqi. Optik. 126, 1433-1438 (2015)

4. L. Bo, H. Shiyong, T. Leena, T. Eino, C. Lulu, D. Rajendra, H. Liisa. Displays. 61 (2020)
5. H. Jin, S.Jin, L. Chen, S. Cen, K. Yuan. IEEE Photonics Journal. 7. 1-1 (2015)

6. D. Lili, Q. Li, X. Wenhai, Z. Lidong. IEEE Photonics Journal. 9. 1-1 (2017)

7. S. Park, G. Kyung, D. Choi, J. Yi, S. Lee, B. Choi. S. Lee. Applied Ergonomics. 78. 26-36 (2019)

8. L. Chaowen, Y. Fengming, W. Bowen, Y. Changhao. Clin Exp Optom. 102. 513-520 (2019) 